Article

\title{
Performance Analysis of IEC 61850 Messages in LTE Communication for Reactive Power Management in Microgrids
}

\author{
S. M. Suhail Hussain ${ }^{1, *(\mathbb{D})}$, Mohd Asim Aftab ${ }^{2}$ and Taha Selim Ustun ${ }^{1}$ (D) \\ 1 Fukushima Renewable Energy Institute (FREA), National Institute of Advanced Industrial Science and \\ Technology (AIST), Koriyama 963-0215, Japan; selim.ustun@aist.go.jp \\ 2 Electrical and Instrumentation Engineering Department, Thapar Institute of Engineering and Technology, \\ Patiala 147001, Punjab, India; asim.aftab@thapar.edu \\ * Correspondence: suhail.hussain@aist.go.jp; Tel.: +81-80-3145-1988
}

Received: 20 October 2020; Accepted: 16 November 2020; Published: 17 November 2020

\begin{abstract}
Reactive power management in microgrids with high penetration of distributed renewable energy sources (DRESs) is challenging. The intermittent generation of DRES makes the power management cumbersome. Generally, Flexible AC Transmission System (FACTS) devices such as Distribution Static Compensator (DSTATCOM) are employed for reactive power compensation in microgrids. However, for effective results in microgrids, coordinated operation between DSTATCOM and Distributed Energy Resources (DERs) is required. In this paper, IEC 61850 communication is proposed for realizing coordinated operation between microgrid controller (MGCC), DSTATCOM and DERs. In microgrids, there may be large number of DERs dispersed over a large area. Hence, the underlying communication network technology for IEC 61850 communication must be highly scalable with wide range. Recently developed communication technology Long Term Evolution (LTE) is a promising solution since it offers high data rates, reliability, scalability and longer range. In this paper, the developed IEC 61850 based reactive power management system is tested with the LTE technology and the performance evaluation tests have been performed. Firstly, IEC 61850 messages have been mapped on LTE stack to enable their transmission. Then, simulations over a network emulator have been performed to evaluate the performance of IEC 61850 communication message exchanges over LTE network in terms of End to End (ETE) delays.
\end{abstract}

Keywords: long term evolution (LTE); IEC 61850; R-GOOSE; R-SV; MMS; microgrid; reactive power management

\section{Introduction}

The growing awareness towards climate change and rapidly depleting fossil fuel sources has led to adaption of distributed and renewable energy sources (DRESs) for power generation [1,2]. The integration of DERs at the distribution system has led to many challenges for the legacy power grids such as bidirectional power flows and issues in protective relay settings [3]. Furthermore, the intermittent power generation by the renewable DRESs makes energy management even more cumbersome. In order to tackle the intermittent power generation by renewable DERs, many solutions have been reported such as microgrids [4] and virtual power plants [5].

The management of intermittent DERs in a microgrid during islanded mode of operation is quite challenging. Since there is no grid support, the reactive power management has to be done with microgrid components. Although smart inverters have the capability to provide reactive power support, still most of the existing grid inverters do not have such capabilities [6]. In order to compensate the 
reactive power, distribution-FACTS devices such as Distribution Static Compensator (DSTATCOM) are deployed in microgrids [7]. A well-coordinated strategy between different DERs, DSTATCOM, loads and microgrid controllers is required to ensure effective reactive power management. This coordination among different components requires a reliable microgrid communication network.

Successful use of these coordinated strategies is hindered by the lack of scalable, reliable and interoperable communication mechanisms. Recently, IEC 61850 standard has been gaining momentum as a promising communication standard for smart grids [8]. Thanks to its object oriented and interoperable information models, IEC 61850 is poised to be a future communication standard for smart grids [9]. IEC 61850 information models for different power utility components are available in literature, e.g., Electric Vehicle (EV) [10], home energy management system [11], energy routers [12], PV stations [13]. Apart from information models, IEC 61850 standards define type of communication messages, namely Generic Object-Oriented Substation Event (GOOSE), Sample Value (SV) and Manufacturing Message Specification (MMS). The structure and underlying protocols for these messages are well defined. However, IEC 61850 standards do not specify any particular technology at physical layer for communication of these messages.

In [14], authors presented IEC 61850 communication model for microgrids to achieve plug and play operation. However, there is no discussion on the details of underlying microgrid communication network. For IEC 61850 based communication in microgrids, switched Ethernet has been largely used. In [15], authors proposed and evaluated the use of different WiFi wireless technologies for IEC 61850 message exchanges. Other wireless technologies such as Wimax [16], Cognitive Radio [17,18] for exchanging IEC 61850 messages in microgrids were explored. However, WiFi and WiMAX technologies have limited range and may not be feasible for microgrid communication networks spread over a large area. Long Term Evolution (LTE) technology is emerging as a promising solution with high coverage range easily satisfying the microgrid communication network requirements. Furthermore, LTE offers very high data rates and system reliability compared to other existing wireless technologies [19].

However, many technical challenges exist which obstruct the adaptation of LTE for IEC 61850 communication. The LTE technology is originally designed for human-centric communications focusing on broadband services [20]. Therefore, it needs to be adopted for smart grid communication by creating smart grid traffic prioritizations. In [21], authors developed an LTE scheduler to classify and prioritize all IEC 61850 messages in LTE network. In [22], authors defined two QoS class Identifiers (QCI) with different priority levels for GOOSE/SV and MMS messages respectively. However, according to IEC 61850-8-1 the GOOSE and SV messages have different QoS priority requirements [23]. In this paper, as per IEC 61850-5 QoS requirements different QCI priority levels are considered for GOOSE, SV and MMS messages. The performance evaluation of IEC 61850 messages in terms of end to end delays for different QoS priority levels is well investigated [21-25]. However, in previous works the performance evaluation is carried out with same QCI priority level for GOOSE and SV messages. In this paper, the performance evaluation in terms of ETE delays with different QCI priority levels of GOOSE and SV in accordance with IEC 61850-5 requirements is presented.

In literature, there is little research on the impact of LTE device to device (D2D) communication on IEC 61850 message exchanges, especially GOOSE and SV. In [22], authors studied the impact of D2D communication on arbitrary GOOSE and SV message exchanges in distribution system. However, the impact of D2D communication is dependent on the proximity of the devices in the network which in turn depends on the system topology and specific message exchanges corresponding to an application. In this paper, the impact of D2D communication on latencies in GOOSE and SV messages exchanged for reactive power management in IEC 61850 based microgrid communication network is reported. Furthermore, this paper presents the demonstration of the reactive power management strategy realized through IEC 61850 message exchanges over LTE network. The simulation results for IEC 61850 message exchanges over LTE network for realizing the reactive power management in microgrid are presented. The contributions of this paper can be summarized as: 
1. IEC 61850 communication model is developed for reactive power management in microgrid.

2. The developed IEC 61850 model is mapped to LTE and adapted for operation

3. Lab-tests have been done for E2E delay performance evaluation of R-GOOSE and R-SV messages over LTE network with D2D communication.

The paper is organized as follows. Section 2 presents a brief overview of reactive power management in microgrids with DSTATCOM. Section 3 details IEC 61850 system modeling and information exchanges for realizing reactive power management in microgrid. Section 4 presents the mapping for IEC 61850 messages to LTE. Section 5 presents network emulator tests and the performance evaluation of IEC 61850 messages over LTE D2D communication network. Finally, conclusions are presented in Section 6.

\section{Overview of Reactive Power Management in Microgrids}

High penetration of DRES in microgrids creates operational problems such as load power balance, voltage regulation and reactive power management. In islanded mode, the reactive power management becomes more cumbersome due to absence of dominant source of generation and fast acting generators. The reactive power management can be achieved by installing a DSTATCOM in microgrid $[7,26]$. Due to intermittency of RES and their oscillatory generation profile, the reactive power demand in microgrid changes which must be compensated dynamically by DSTATCOM. This is achieved by providing appropriate reference AC voltage setting in the DSTATCOM controller to provide required reactive power.

The reference AC voltage signal developed in [20] is given by Equation (1)

$$
\begin{gathered}
v_{\text {acref }}=v_{0}-m Q_{\text {STATCOM }}+k_{1} \frac{R_{1} P_{1}+X_{1} Q_{1}}{V_{11} V_{22}}\left[S_{1}\right]+k_{2} \frac{R_{2} P_{2}+X_{2} Q_{2}}{V_{22} V_{33}}\left[S_{2}\right]+\ldots+k_{i} \frac{R_{i} P_{i}+X_{i} Q_{i}}{V_{i i} V_{i+1, i+1}}\left[S_{i}\right] \\
+\ldots+k_{n} \frac{R_{n} P_{n}+X_{n} Q_{n} V_{n n} V_{n+1, n+1}}{V_{n}}\left[S_{n}\right]
\end{gathered}
$$

where $v_{o}$ is nominal value of voltage, $m$ is controller gain, $Q_{S T A T C O M}$ is reactive power injection capacity limit of DSTATCOM, $k$ is the proportionality factor introduced to provide maximum reactive power compensation and $[S]$ is the status matrix populated on the status of a DER in microgrid. Based on DER status the matrix $S$ is given by Equation (2)

$$
[S]=\left[\begin{array}{llllllllll}
1 & 0 & 0 & \ldots & . & 1 & 1 & \ldots & 0
\end{array}\right]^{T} 1 x p
$$

where $p$ is the number of DERs in the microgrid. The Microgrid Central Communication Controller (MGCC) populates the status matrix and provides appropriate settings to DSTATCOM controller for reactive power management.

For dynamic reactive power management in microgrid, the DSTATCOM must have a system wide information of DERs. Thus, communication among various microgrid entities such as DSTATCOM, DERs and MGCC is essential. Due to the advantages discussed earlier, IEC 61850 based communication has been adopted to model reactive power management as discussed in the next section.

\section{IEC 61850 Based Information Modeling and Mapping of Reactive Power Management in Microgrids}

For enabling IEC 61850 based communication in microgrid, its components such as DERs and loads must be designed in accordance with IEC 61850 standard. IEC 61850 based communication design requires communication configurations which includes information modeling of DERs, service modeling, mapping to communication protocols and telecommunication media [8]. Information modelling can be defined as a method of providing standardized syntax, semantics and hierarchical structures to the data that is exchanged among different devices and systems [8]. As per IEC 61850, the information modeling is achieved by defining logical nodes (LNs) and data objects (DOs). LNs are a group of DOs which serve specific function and are defined conceptually in IEC 61850 standard. 
Modeling of microgrid components such as DERs is developed in IEC 61850-7-420 [27]. For modeling DSTATCOM controller, the information model developed in [7] has been considered in this paper.

\subsection{Information Modeling of DSTATCOM Controller}

IEC 61850 based information modeling for DSTATCOM controller is realized using pre-existing logical nodes and new logical nodes developed in [7]. The information model for DSTATCOM controller is shown in Figure 1.

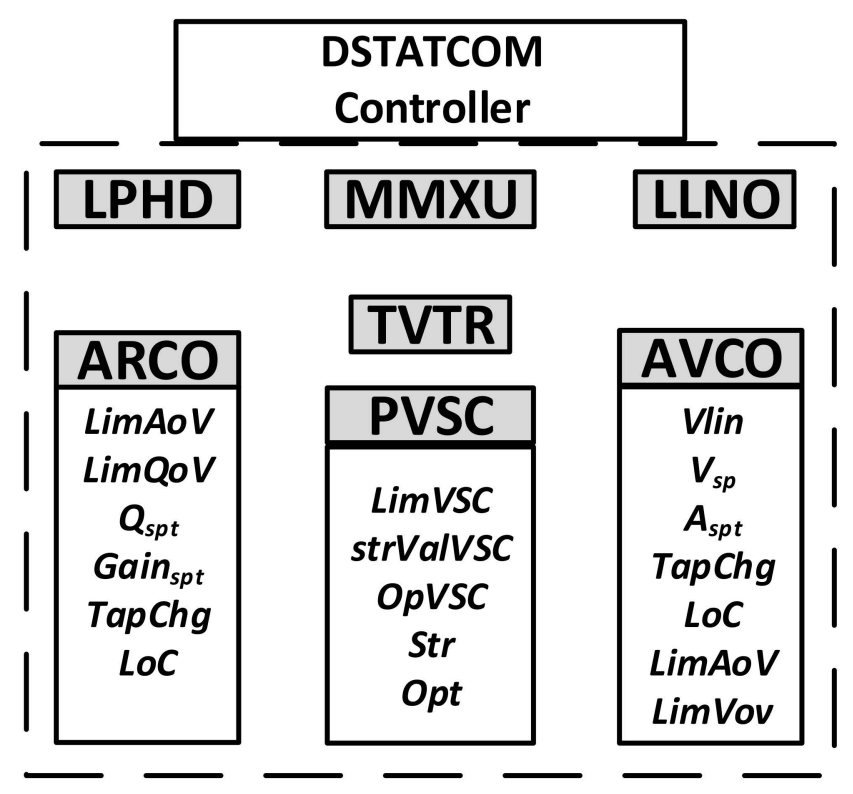

Figure 1. IEC 61850 based information model of DSTATCOM controller [7].

LNs LPHD and LLNO provide information about the physical device such as name plate ratings. LN MMXU is used for providing metering and measurement information. LN TVTR is for modeling of potential transformer (PT). LN ARCO is used to provide reactive power compensation. It has various DOs such as "LimAoV" which specifies the limit of operation for current, "LimQoV" which specifies limit of operation for reactive power. DO "Qspt" is used as set point for reactive power required and "Gainspt" is the gain of the controller. DO "TapChg" is used for changing reactive power supply while $\mathrm{DO}$ " $\mathrm{LoC}$ " is for local operation.

LN AVCO is for automatic voltage control and has following DOs, "Vlin" which takes voltage value from the LN TVTR, "LimAoV" and "LimVoV" which provides the current and voltage operating limits respectively, "Aspt", "Vspt" which are the set points for current and voltage respectively. The LN PVSC is for protection of Voltage Source Converter (VSC) and contains DOs such as "LimVSC" for operating limits of VSC, "StrValVSC" for preventing any overloads, "OpVSC" for safe operating range and "Str" and "Opt" are for starting and operating the device.

\subsection{IEC 61850 Information Exchanges for Reactive Power Management in Test Microgrid}

IEC 61850 enabled reactive power compensation scheme using DSTATCOM has been applied on a test microgrid as shown in Figure 2. The test microgrid consists of four DERs viz. PV power plant, wind power plant (WPP), Diesel plant and a battery system and loads. The DSTATCOM has been placed on bus 4 and 5 for reactive power support as per the requirement at load bus. The IEC 61850 information model of different components of microgrid with their respective LNs for the test microgrid considered in Figure 2 is shown in Figure 3. The IEC 61850 based messages flows have been developed in this section. 


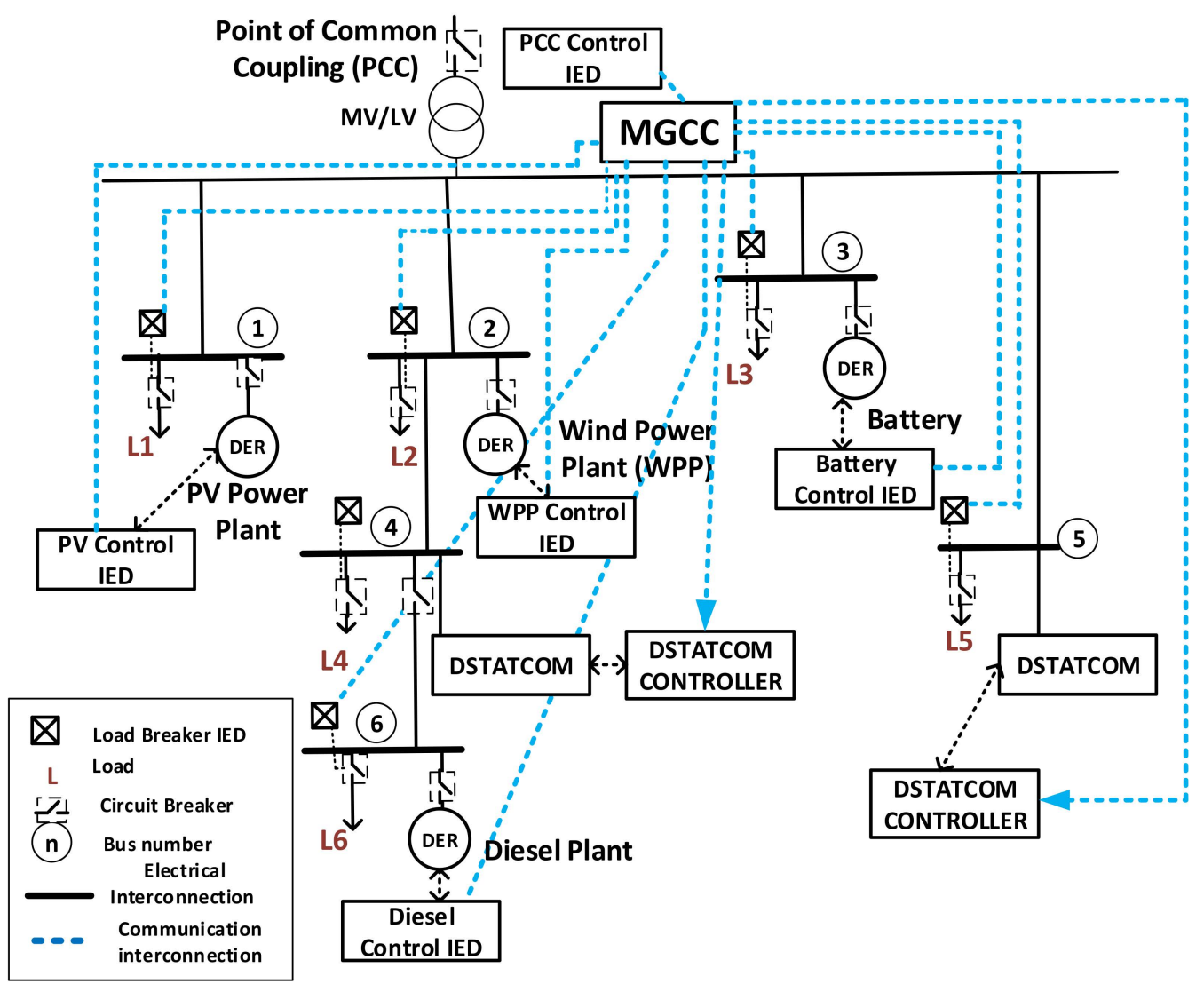

Figure 2. Microgrid test system.

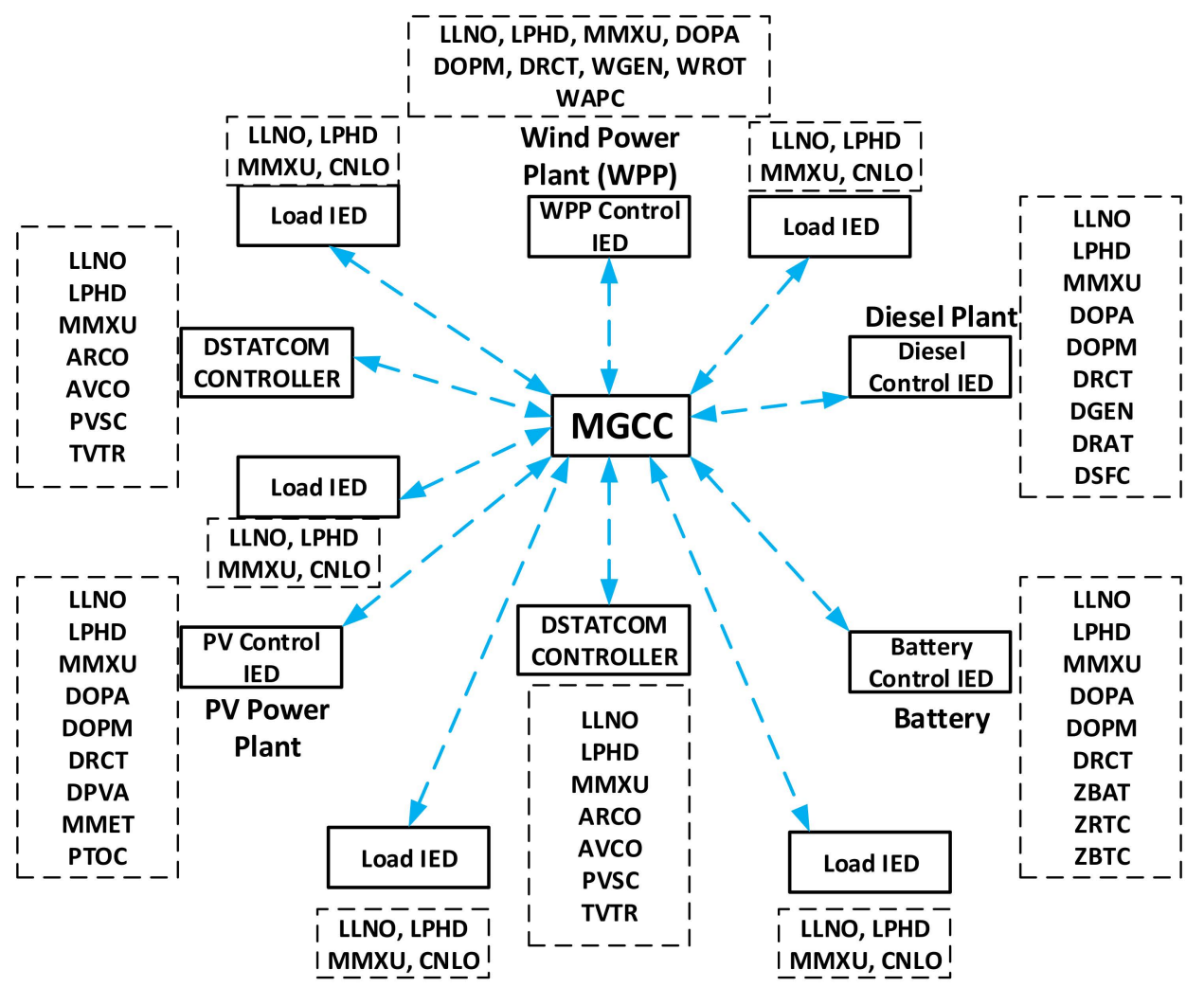

Figure 3. IEC 61850 communication model of test microgrid. 
A scenario has been considered and IEC 61850 information exchanges have been developed in this section. The microgrid is operating in grid connected mode. A fault develops in the microgrid primary bus and it is isolated from the main grid. This is done by disconnecting the microgrid at Point of Common Coupling (PCC). The MGCC issues a trip command to Circuit Breaker (CB) in PCC control Intelligent Electronic Device (IED). This sets the DO "BlkOpn" of the LN XCBR to 0 for opening the CB block. The information flow is as per Equation (3).

$$
\text { MGCC } \rightarrow \text { PCC_ControlIED \$XCBR \$DPC\$ Pos }
$$

Once the fault is cleared, the microgrid operates in islanded mode to meet its load demand through the available generation capacity. The Diesel generator acts as a dominant generator. In this scenario, it is assumed that all the DERs are operating and the load demand of the microgrid is met in islanded mode. The status of all the DERs is updated to the MGCC through the DER control IED. The MGCC, then, populates the status matrix $S$ defined in Equation (2). The information flow for PV power plant and wind power plant conveying the status to MGCC is given as Equation (4)

$$
\begin{aligned}
& \text { PV_ControlIED \$DPST \$ST \$ECPConn } \rightarrow \text { MGCC } \\
& \text { WPP_ControllED\$DPST \$ST \$ECPConn } \rightarrow \text { MGCC }
\end{aligned}
$$

Similarly, other DERs also update their status to MGCC. The MGCC populates the status vector S. In addition, after fetching the demand, the MGCC computes the operating modes of DER such as constant Watts, constant VARs and communicates them to the DERs. The information flow of the PV power plant is shown in Equation (5)

$$
\text { MGCC } \rightarrow \text { PV_ControlIED \$DOPA \$ERY \$DEROpMode }
$$

Due to a sudden increase in demand, there is a voltage dip in the microgrid. This is to be compensated by the DSTATCOM by injecting reactive power into the microgrid. The MGCC computes the required voltage and provides the set point for the reactive power to the DSTATCOM controller as per Equation (6)

$$
\begin{aligned}
\text { MGCC } & \rightarrow \text { DSTATCOM_ControllerIED1 } \$ \text { ARCO } \$ S P \$ Q s p t \\
\text { MGCC } & \rightarrow \text { DSTATCOM_ControllerIED2 } \$ \text { ARCO \$SP \$Qspt }
\end{aligned}
$$

The active and reactive powers of the DERs which are computed using DOs "TotW," "TotVAr" and "PhV" of the MMXU logical node of the DER control IED. For different DERs this updated in different communication flows. For Diesel generator, this information flow is as given in Equation (7),

$$
\text { Diesel_ControlIED \$MMXU \$SP \$TotW \&TotVAr \&PhV } \rightarrow \text { MGCC }
$$

Based on these values, the required reference ac voltage is computed by the MGCC and is updated to the DSTATCOM controllers by the information flow as given in Equation (8). There might be a scenario when the requirement of reactive power is less and can be compensated by a single DSTATCOM and in that case only one DSTATCOM functions.

$$
\begin{aligned}
& \text { MGCC } \rightarrow \text { DSTATCOM_ControllerIED1 \$AVCO \$SP \$Vsp } \\
& \text { MGCC } \rightarrow \text { DSTATCOM_ControllerIED2 \$AVCO \$SP \$Vsp }
\end{aligned}
$$

Over the established communication flows, the required reactive power support is met by the DSTATCOM in islanded microgrid. The local merging unit (MU) IEDs located along with the control IEDs of different DERs or DSTATCOM controller, continuously send measured voltage and current values to their respective control IEDs as R-SV messages. The IEC 61850 based information exchanges and their mapping to the appropriate IEC 61850 services are presented in Table 1. 
Table 1. Message exchanges for Reactive Power Management in Microgrid.

\begin{tabular}{|c|c|c|c|c|}
\hline \multicolumn{2}{|c|}{ Message Exchanges for Reactive Power Management } & \multirow{2}{*}{ ACSI Service } & \multirow{2}{*}{ SCSM } & \multirow{2}{*}{ IEC 61850 Message Type } \\
\hline Source IED & Destination IED & & & \\
\hline MGCC & PCC_ControlIED $\rightarrow$ XCBR $\rightarrow$ Pos $\rightarrow{ }^{\prime} 0^{\prime}$ & SendGOOSEMessage & GoosePDU & R-GOOSE \\
\hline PV_ControlIED $\rightarrow$ DPST $\rightarrow$ ECPConn & MGCC & GetDataValues & $\begin{array}{l}\text { Read-MMS Request } \\
\text { Read-MMS Response }\end{array}$ & MMS \\
\hline WPP_ControlIED $\rightarrow$ DPST $\rightarrow$ ECPConn & MGCC & GetDataValues & $\begin{array}{l}\text { Read-MMS Request } \\
\text { Read-MMS Response }\end{array}$ & MMS \\
\hline Battery_ControlIED $\rightarrow$ DPST $\rightarrow$ ECPConn & MGCC & GetDataValues & $\begin{array}{l}\text { Read-MMS Request } \\
\text { Read-MMS Response }\end{array}$ & MMS \\
\hline Diesel_ControlIED $\rightarrow$ DPST $\rightarrow$ ECPConn & MGCC & GetDataValues & $\begin{array}{l}\text { Read-MMS Request } \\
\text { Read-MMS Response }\end{array}$ & MMS \\
\hline MGCC & PV_ControlIED $\rightarrow$ DOPA $\rightarrow$ DEROpMode & SendGOOSEMessage & GoosePDU & R-GOOSE \\
\hline MGCC & Diesel_ControlIED $\rightarrow$ DOPA $\rightarrow$ DEROpMode & SendGOOSEMessage & GoosePDU & R-GOOSE \\
\hline MGCC & WPP_ControlIED $\rightarrow$ DOPA $\rightarrow$ DEROpMode & SendGOOSEMessage & GoosePDU & R-GOOSE \\
\hline MGCC & DSTATCOM_ControllerIED1 $\rightarrow$ ARCO $\rightarrow$ Qspt & SendGOOSEMessage & GoosePDU & R-GOOSE \\
\hline MGCC & DSTATCOM_ControllerIED2 $\rightarrow$ ARCO $\rightarrow$ Qspt & SendGOOSEMessage & GoosePDU & R-GOOSE \\
\hline PV_ControlIED $\rightarrow$ MMXU $\rightarrow$ TotW, TotVAr, PhV & MGCC & GetDataValues & $\begin{array}{l}\text { Read-MMS Request } \\
\text { Read-MMS Response }\end{array}$ & MMS \\
\hline WPP_ControlIED $\rightarrow$ MMXU $\rightarrow$ TotW, TotVAr, PhV & MGCC & GetDataValues & $\begin{array}{l}\text { Read-MMS Request } \\
\text { Read-MMS Response }\end{array}$ & MMS \\
\hline Diesel_ControlIED $\rightarrow$ MMXU $\rightarrow$ TotW, TotVAr, PhV & MGCC & GetDataValues & $\begin{array}{l}\text { Read-MMS Request } \\
\text { Read-MMS Response }\end{array}$ & MMS \\
\hline Battery_ControlIED' $\rightarrow$ MMXU $\rightarrow$ TotW, TotVAr, PhV & MGCC & GetDataValues & $\begin{array}{l}\text { Read-MMS Request } \\
\text { Read-MMS Response }\end{array}$ & MMS \\
\hline MGCC & DSTATCOM_ControllerIED1 $\rightarrow$ AVCO $\rightarrow V s p$ & SendGOOSEMessage & GoosePDU & R-GOOSE \\
\hline MGCC & DSTATCOM_ControllerIED1 $\rightarrow$ AVCO $\rightarrow V s p$ & SendGOOSEMessage & GoosePDU & R-GOOSE \\
\hline All MU_IED & All Control IEDs & SendSVMessage & SvPDU & R-SV \\
\hline
\end{tabular}




\section{Mapping of IEC 61850 Messages to LTE}

\subsection{Overview of Long Term Evolution (LTE)}

LTE is 4th generation of wireless mobile communication technology. LTE provides high data rates, low latencies and packet optimized radio access technologies with flexible bandwidth deployments. LTE uses Orthogonal Frequency Division Multiple Access (OFDMA) and Multiple Input and Multiple Output (MIMO) at physical layers for flexible resource allocations. It operates in bandwidth range of $1.4 \mathrm{MHz}$ to $20 \mathrm{MHz}$.

The LTE communication network consists of three main components: User Equipment (UE), eNodeB (base station) and MME. The eNodeB is a core component of LTE which performs all the tasks related to radio resource sharing and retransmission protocols, etc.

The Quality of Service (QoS) in LTE is designed based on the data flows and bearers. The bearer is a set of QoS requirements indicated by QoS Class Identifier (QCI). The QCI for each service type is defined by different parameters such as priority level, packet delay, packet error rate and guaranteed bit rate. Table 2 lists different QCI classes defined for LTE. From Table 2 it can be observed that all the QCI classes are oriented for human-based services such as voice/video conversation, etc. These defined classes do not match the requirement of IEC 61850 smart grid communication message exchanges.

Table 2. QoS class Identifiers (QCI) characteristics of Long Term Evolution (LTE) networks.

\begin{tabular}{ccccc}
\hline QCI & Priority Level & Packet Delay $(\mathbf{m s})$ & Packet Error Loss Rate & Service Type \\
\hline 1 & 2 & 100 & $10^{-2}$ & Conversation Voice \\
\hline 2 & 4 & 150 & $10^{-3}$ & Conversation Video \\
\hline 3 & 3 & 50 & $10^{-3}$ & Real-time gaming \\
\hline 4 & 5 & 300 & $10^{-6}$ & Non-Conversation Video \\
\hline 65 & 0.7 & 75 & $10^{-2}$ & Mission critical PTT \\
\hline 66 & 2 & 100 & $10^{-2}$ & Non-Mission critical PTT \\
\hline 5 & 1 & 100 & $10^{-6}$ & IMS signaling \\
\hline 6 & 6 & 100 & $10^{-6}$ & Video TCP-based \\
\hline 7 & 7 & 300 & $10^{-3}$ & Real-time \\
\hline 8 & 8 & 100 & $10^{-6}$ & Video TCP-based \\
\hline 9 & 9 & 300 & $10^{-6}$ & Video TCP-based \\
\hline 69 & 0.5 & 60 & $10^{-6}$ & Mission critical live \\
\hline 70 & 5.5 & 200 & $10^{-6}$ & Mission critical live \\
\hline 10 & 0.1 & 10 & $10^{-6}$ & IEC 61850 GOOSE/R-GOOSE \\
\hline 11 & 0.5 & 10 & $10^{-6}$ & IEC 61850 SV/ R-SV \\
\hline 12 & 1 & 200 & $10^{-6}$ & \\
\hline & & & & \\
\hline
\end{tabular}

Hence, similar to [23], this paper proposes to create three QoS classes to support GOOSE / R-GOOSE, SV / R-SV and MMS messages, respectively. The Different QCI characteristics of LTE including the newly defined QCIs are shown in Table 2. The R-GOOSE message which carries the time critical commands is given the highest priority with 0.1 priority level. The R-SV and MMS messages are given 0.5 and 1 priority level, respectively.

\subsection{Mapping of IEC 61850 Messages to LTE}

IEC 61850 standard specifies the set of protocols and stack for network, transport, session, presentation and application layers for MMS, R-GOOSE and R-SV messages. However, the standards 
do not stipulate any specific protocol for data link and physical layers. MMS message uses TCP/IP for transport and network layers whereas R-GOOSE/R-SV messages use UDP/IP stack.

Figure 4 gives the details of different protocols stipulated for MMS, R-GOOSE and R-SV messages at presentation, session and application layers. MMS, R-GOOSE and R-SV stack is mapped to the data link and physical layer stack of LTE as shown in Figure 4. Hence, with the complete stacks as shown in Figure 4, the MMS, R-GOOSE and R-SV messages can operate over LTE systems.

OSI Layers
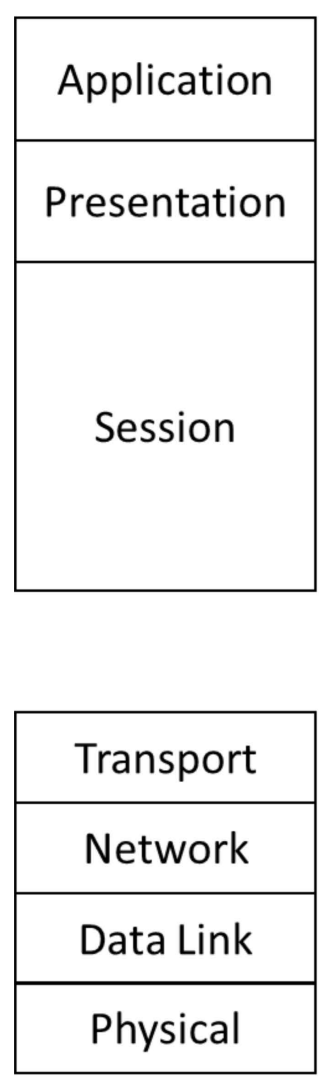

MMS STACK

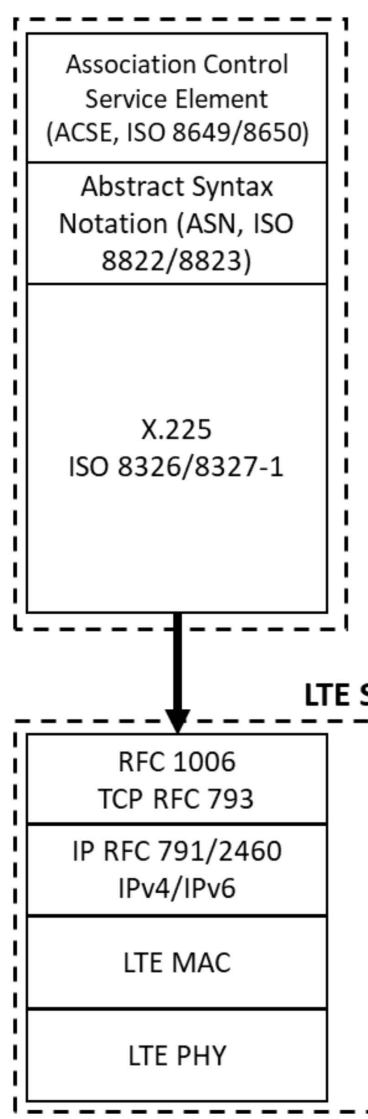

R-GOOSE / R-SV STACK
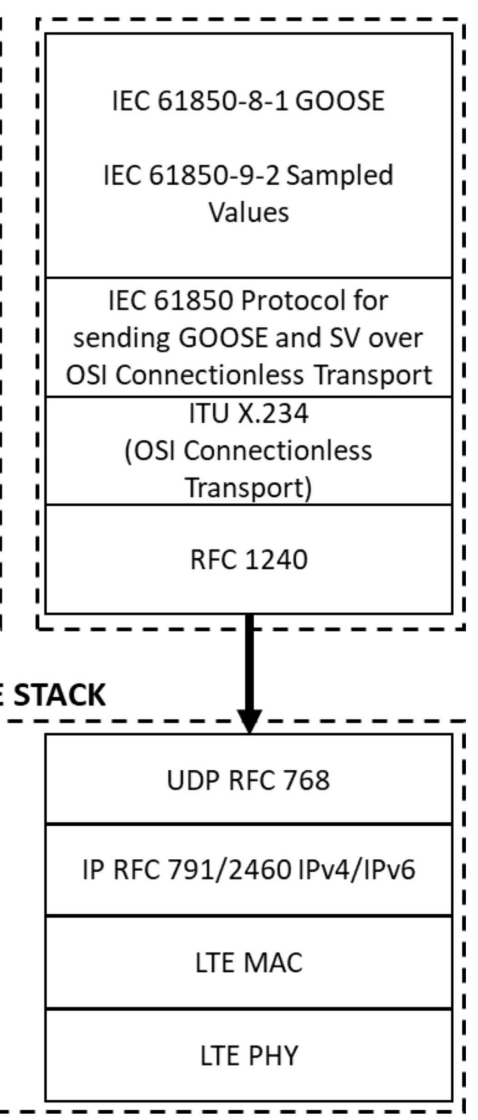

Figure 4. Mapping of Manufacturing Message Specification (MMS) and R-GOOSE messages over LTE.

\section{Performance Evaluation of IEC 61850 Message Transmission over LTE}

In order to evaluate the performance of IEC 61850 messages under LTE technology, simulation of microgrid communication network is carried out in NetSim simulator software tool. Figure 5 shows the simulation of LTE communication network for microgrid test system in Figure 2. For simulation, it is assumed that the microgrid is spread over an area of $2 \mathrm{~km} \times 2 \mathrm{~km}$ and contains 3 eNodeB. The simulation parameters considered for the NetSim simulation pertaining to LTE are given in Table 3.

The traffic is configured in the simulation as per Table 1 between different source and destination IEDs using the "Application" module in NetSim software tool. The average End to End (ETE) delays obtained for different message exchanges for realizing reactive power management are shown in Table 4. From Table 4, it is quite clear that the ETE delays for R-SV messages are in 1.5-1.9 ms range for the considered test microgrid, which is within the acceptable range of $10 \mathrm{~ms}$ limit for R-SV messages. Similarly, the R-GOOSE messages have maximum of 1.02 ms delay, which is well below the $3 \mathrm{~ms}$ acceptable range. The MMS messages have around $3.5 \mathrm{~ms}$ delays, which is quite acceptable. Furthermore, the packet loss rates for different IEC 61850 message exchanges are also shown in Table 4 . It can be noticed that the packet loss rate is below the $10^{-6}$ limit, as specified by the standards. 


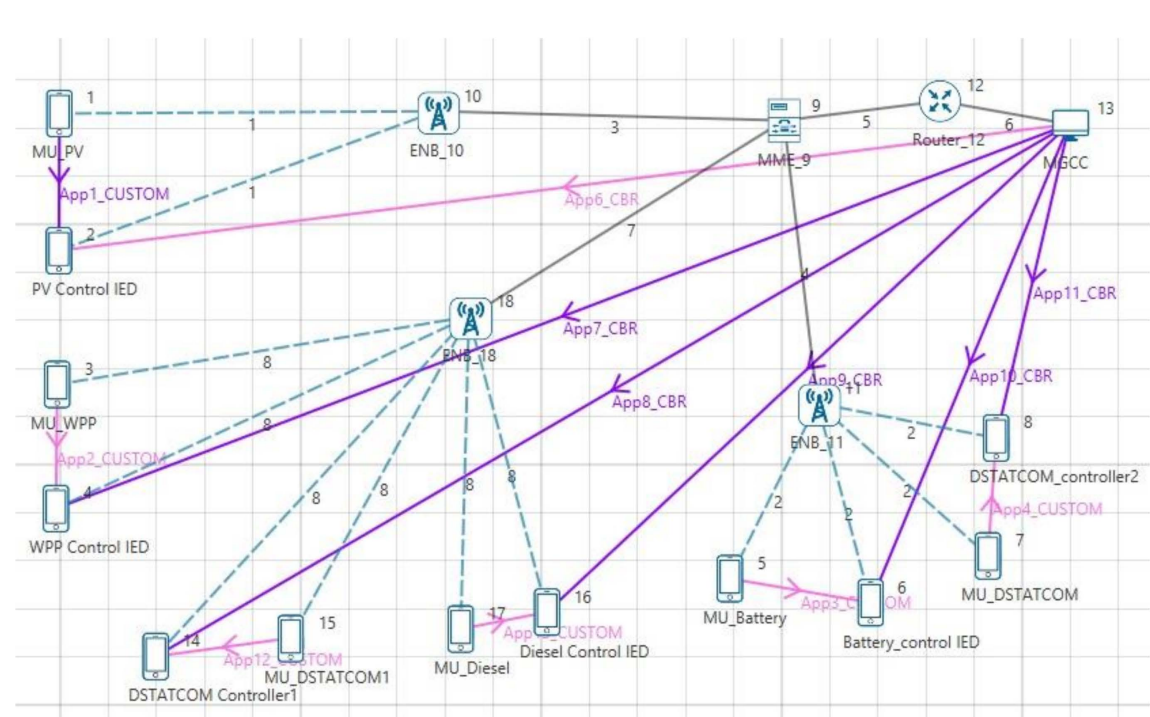

Figure 5. Simulated Model of LTE communication network of microgrid test system.

Table 3. LTE Communication Network Simulation Parameters.

\begin{tabular}{cc}
\hline Parameter & Value \\
\hline Bandwidth & $15 \mathrm{MHz}$ \\
\hline Transmission Time Interval (TTI) & $1 \mathrm{~ms}$ \\
\hline Modulation & $64 \mathrm{QAM}$ \\
\hline DL / UL access scheme & OFDMA / SC-FDMA \\
\hline FFT size & 1536 \\
\hline Carrier Aggregation & Intra band Contiguous \\
\hline Frame / Sub-Frame / Slot duration & $10 / 1 / 0.5 \mathrm{~ms}$ \\
\hline Subcarrier spacing (kHz) & 15 \\
\hline eNodeB, User & 3,12 \\
\hline
\end{tabular}

Table 4. End to End (ETE) Delays for Different Messages in Microgrid for Reactive Power Management.

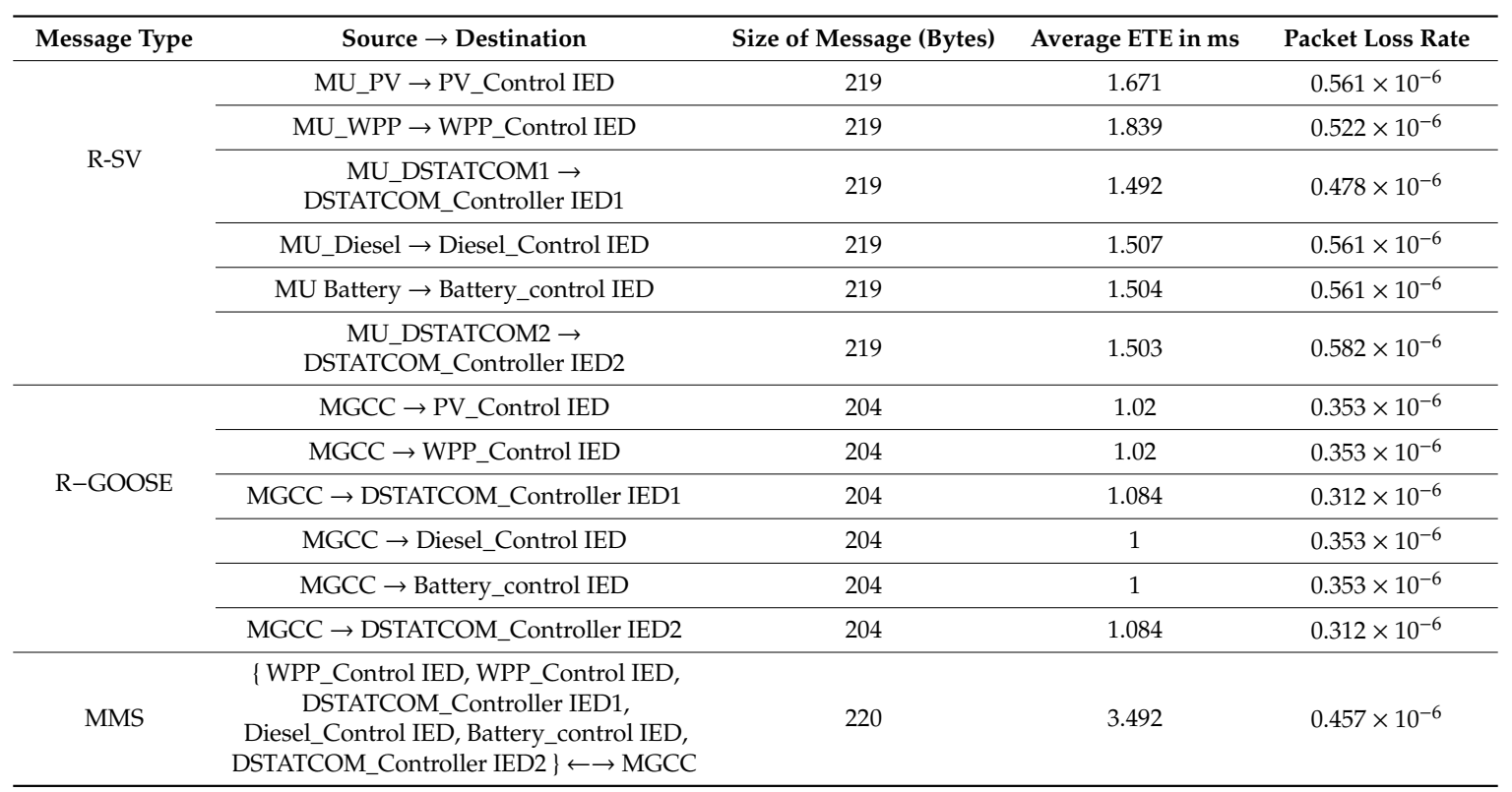


The end devices in LTE network have the capability to establish a direct device to device (D2D) communication without involving eNodeB. This D2D communication between close proximity devices helps reduce burden on eNodeB and thereby increases its throughput and data rates.

The R-SV message exchanges between different MU IEDs and respective control IEDs are set up using LTE D2D communication. When D2D communication is enabled, the R-SV traffic is directly exchanged between end devices and thus reduces the processing burden on eNodeB. When, the D2D communication is disabled, the R-SV traffic reaches the destination control IED via the eNodeB which increases the overall ETE delay. Figure 6 shows the ETE delays of R-SV message exchanged between MU_Battery and Battery_control IED when D2D communication is enabled and disabled.

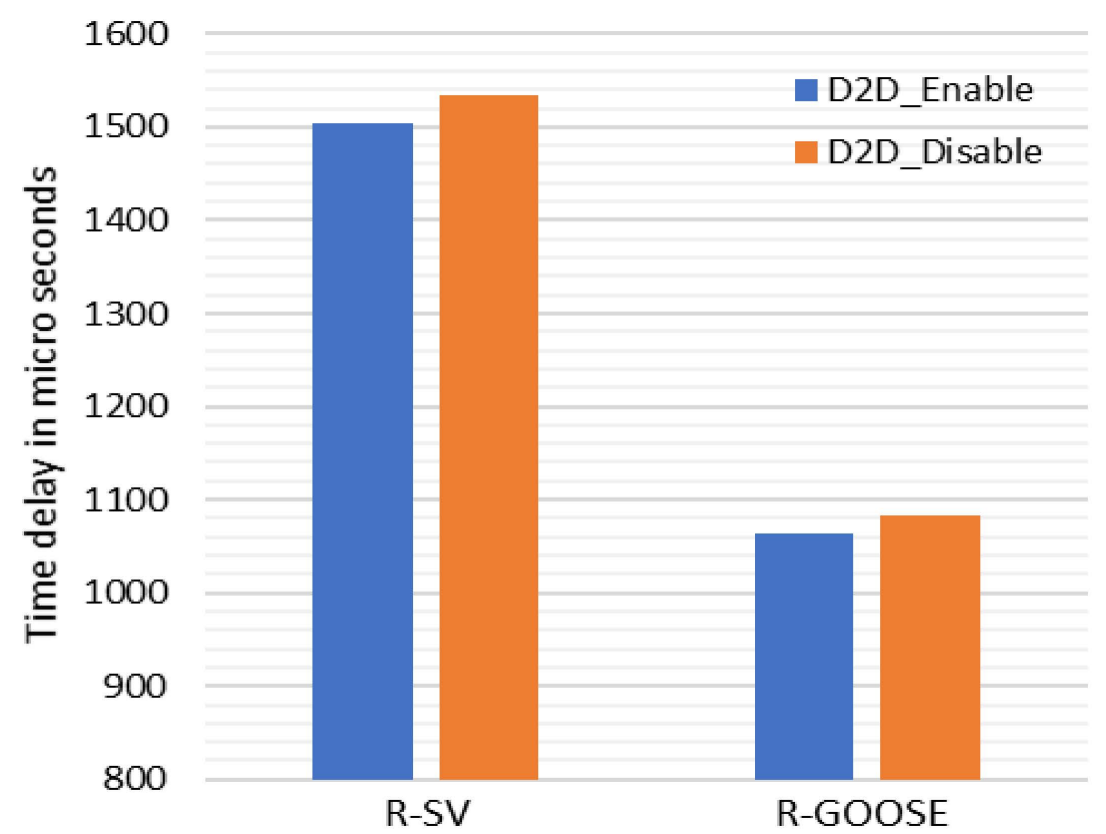

Figure 6. ETE delay of R-SV and R-GOOSE message when device to device (D2D) communication in LTE is enabled and disabled.

In the considered test microgrid system communication network, the DSTATCOM_ControllerIED1 is in close proximity to MGCC. Hence, D2D communication between these IEDs for exchanging R-GOOSE messages is established. From Table 4 it is already evident that the ETE delays for R-GOOSE message exchanged between MGCC and DSTATCOM_ControllerIED1 when D2D is disabled is $1.084 \mathrm{~ms}$. When D2D is enabled, the ETE delay obtained for R-GOOSE message exchange is found out to be $1.065 \mathrm{~ms}$.

\section{Conclusions}

In this paper, reactive power management in a microgrid is realized through IEC 61850 communication message exchanges over an LTE network. The mapping of IEC 61850 messages R-GOOSE, R-SV and MMS to the LTE stack is presented. Furthermore, the QCI classes prioritizing the R-GOOSE, R-SV messages are developed. LTE network simulations are performed using network simulator tools to estimate the ETE delays for different IEC 61850 message exchanges in a microgrid. From the simulation results it is concluded that the ETE delays of IEC 61850 message exchanges for realizing reactive power management are well within the limits set by standards. Hence, the LTE technology can be successfully employed for IEC 61850 microgrid communication networks. These results mean that with LTE technology, IEC 61850-based communication networks can be implemented for microgrids where devices can be spread over a large area. This is important for overcoming limitations of other communication technologies such as Wi-Fi and WiMAX. 
Author Contributions: Conceptualization, S.M.S.H., M.A.A. and T.S.U.; methodology, S.M.S.H. and M.A.A.; software, S.M.S.H.; validation, S.M.S.H., M.A.A.; formal analysis, S.M.S.H., M.A.A. and T.S.U.; investigation, S.M.S.H., M.A.A.; resources, S.M.S.H., M.A.A. and T.S.U.; writing-original draft preparation, S.M.S.H., M.A.A.; writing-review and editing, S.M.S.H. and T.S.U.; visualization, S.M.S.H. and M.A.A.; funding acquisition, S.M.S.H. and T.S.U.; All authors have read and agreed to the published version of the manuscript.

Funding: This research was funded by Fukushima Prefectures' Reconstruction Grant, 2020.

Conflicts of Interest: The authors declare no conflict of interest.

\section{References}

1. Javed, K.; Ashfaq, H.; Singh, R.; Hussain, S.M.; Ustun, T.S. Design and Performance Analysis of a Stand-alone PV System with Hybrid Energy Storage for Rural India. Electronics 2019, 8, 952. [CrossRef]

2. Hubble, A.; Ustun, T.S. Scaling renewable energy based microgrids in underserved communities: Latin America, South Asia, and Sub-Saharan Africa. In Proceedings of the 2016 IEEE PES Power Africa, Livingstone, UK, 28 June-3 July 2016; pp. 134-138.

3. Ustun, T.S. Design and Development of a Communication-Assisted Microgrid Protection System. Ph.D. Thesis, Victoria University, Melbourne, Australia, 2013.

4. Hatziargyriou, N. The microgrids concept. In Microgrids: Architectures and Control; John Wiley \& Sons: New York, NY, USA, 2014; pp. 1-24.

5. Nadeem, F.; Aftab, M.A.; Hussain, S.M.; Ali, I.; Tiwari, P.K.; Goswami, A.K.; Ustun, T.S. Virtual power plant management in smart grids with XMPP based IEC 61850 communication. Energies 2019, 12, 2398. [CrossRef]

6. Ustun, T.S.; Aoto, Y. Analysis of smart inverter's impact on the distribution network operation. IEEE AccesS 2019, 7, 9790-9804. [CrossRef]

7. Hussain, S.M.S.; Aftab, M.A.; Ali, I. IEC 61850 modeling of DSTATCOM and XMPP communication for reactive power management in microgrids. IEEE Syst. J. 2018, 12, 3215-3225. [CrossRef]

8. Aftab, M.A.; Hussain, S.M.S.; Ali, I.; Ustun, T.S. IEC 61850 based substation automation system: A survey. Int. J. Electr. Power Energy Syst. 2020, 120, 106008. [CrossRef]

9. Ali, I.; Hussain, S.S. Control and management of distribution system with integrated DERs via IEC 61850 based communication. Eng. Sci. Technol. Int. J. 2017, 20, 956-964. [CrossRef]

10. Aftab, M.A.; Hussain, S.M.S.; Ali, I.; Ustun, T.S. IEC 61850-based communication layer modeling for electric vehicles: Electric vehicle charging and discharging processes based on the international electrotechnical commission 61850 standard and its extensions. IEEE Ind. Electron. Mag. 2020, 14, 4-14. [CrossRef]

11. Ustun, T.S.; Hussain, S.M.S. Standardized communication model for home energy management system. IEEE Access 2020, 8, 180067-180075. [CrossRef]

12. Hussain, S.M.S.; Aftab, M.A.; Nadeem, F.; Ali, I.; Ustun, T.S. Optimal energy routing in microgrids with IEC 61850 based energy routers. IEEE Trans. Ind. Electron. 2020, 67, 5161-5169. [CrossRef]

13. Liu, C.H.; Gu, J.C. Modeling and integrating PV stations into IEC 61850 XMPP intelligent edge computing gateway. Energies 2019, 12, 1442. [CrossRef]

14. Deng, W.; Pei, W.; Shen, Z.; Zhao, Z.; Qu, H. Adaptive micro-grid operation based on IEC 61850. Energies 2015, 8, 4455-4475. [CrossRef]

15. Parikh, P.P.; Sidhu, T.S.; Shami, A. A Comprehensive investigation of wireless LAN for IEC 61850-based smart distribution substation applications. IEEE Trans. Ind. Inform. 2013, 9, 1466-1476. [CrossRef]

16. Hussain, S.M.S.; Tak, A.; Ustun, T.S.; Ali, I. Communication modeling of solar home system and smart meter in smart grids. IEEE Access 2018, 6, 16985-16996. [CrossRef]

17. Hussain, S.M.S.; Aftab, M.A.; Ali, I.; Ustun, T.S. IEC 61850 based energy management system using plug-in electric vehicles and distributed generators during emergencies. Int. J. Electr. Power Energy Syst. 2020, 119, 105873. [CrossRef]

18. Haj-Ahmed, M.A.; Hawa, M.; Shamlawi, L.A.; Alnaser, S.; Alsmadi, Y.M.; Abualnadi, D. Cognitive radio based backup protection scheme for smart grid applications. IEEE Access 2020, 8, 71866-71879. [CrossRef]

19. Dahlman, E.; Parkvall, S.; Sköld, J. 4G LTE/LTE-Advanced for Mobile Broadband; Elsevier, Academic Press: Amsterdam, The Netherlands, 2011.

20. Dahlman, E.; Parkvall, S.; Skold, J.; Beming, P. 3 G Evolution: HSPA and LTE for Mobile Broadband; Elsevier: Amsterdam, The Netherlands, 2007. 
21. Kalalas, C.; Gkatzikis, L.; Fischione, C.; Ljungberg, P.; Alonso-Zarate, J. Enabling IEC 61850 communication services over public LTE infrastructure. In Proceedings of the 2016 IEEE International Conference on Communications (ICC), IEEE, Kuala Lumpur, Malaysia, 22-27 May 2016; pp. 1-6.

22. Mouftah, H.T.; Erol-Kantarci, M.; Rehmani, M.H. LTE-D2D Communication for power distribution grid: Resource allocation for time-critical applications. In Transportation and Power Grid in Smart Cities: Communication Networks and Services; John Wiley \& Sons: New York, NY, USA, 2019; pp. 21-67.

23. Code, P.; Prix, C. Communication networks and systems for power utility automation-Part 8-1: Specific Communication Service Mapping (SCSM)_Mappings to MMS (ISO 9506-1 and ISO 9506-2) and to ISO/IEC 8802-3; International Electrotechnical Commission: Geneva, Switzerland, 2011.

24. Patel, D.; Mohamed, M.I.N.; Mehdi, S.Z.R.; Williams, F.; Sadu, A.; Ponci, F.; Monti, A. Investigating the performance of QoS enabled LTE networks for IEC 61850 based smart grid applications. In Proceedings of the 2016 IEEE International Energy Conference (ENERGYCON), Leuven, Belgium, 4-8 April 2016.

25. Bag, G.; Johansson, M.; Lednicki, L.; Neander, J.; Eriksson, L.; Bogati, R.; Hovila, P.; Saarinen, J.; Torsner, J. Performance evaluation of IEC 61850-90-5 over a latency optimized 3GPP LTE Network. In Proceedings of the 2018 IEEE International Conference on Communications, Control, and Computing Technologies for Smart Grids (Smart Grid Comm), Aalborg, Denmark, 29-31 October 2018.

26. Majumder, R. Reactive power compensation in single-phase operation of microgrid. IEEE Trans. Ind. Electron. 2013, 60, 1403-1416. [CrossRef]

27. Communication Networks and Systems for Power Utility Automation-Part 7-420: Basic Communication Structure-Distributed Energy Resources Logical Nodes; International Electrotechnical Commission: Geneva, Switzerland, 2009.

Publisher's Note: MDPI stays neutral with regard to jurisdictional claims in published maps and institutional affiliations. 